\title{
Anfibios y reptiles asociados a tres especies de bromelias de tanque en el Parque Nacional Guanahacabibes, Cuba
}

\author{
Alfredo García-González ${ }^{1}$, Lázaro Yusnaviel García Padrón², Freddy Delgado Fernández² \& \\ Frander Brian Riverón-Giró ${ }^{1}$ \\ 1. El Colegio de la Frontera Sur (ECOSUR), Unidad Tapachula. Carretera Antiguo Aeropuerto km 2.5, Apartado Postal 36, Tapachula, Chiapas, \\ México. C.P. 30700; alfredmx22@gmail.com; franderb29@gmail.com \\ 2. Centro de Investigaciones y Servicios Ambientales (ECOVIDA). Carretera a Luís Lazo km 2.5, Pinar del Río, Cuba; yusnaviel@gmail.com; \\ freddy@ecovida.vega.inf.cu
}

Recibido 10-IX-2013 • Corregido 11-12-2013 • Aceptado 28-I-2014

\begin{abstract}
Amphibians and reptiles associated to three tank bromeliad species at Guanahacabibes National Park, Cuba. The herpetological fauna associated to three species of tank bromeliads was studied in a semideciduous dry forest on naked karst in the Guanahacabibes National Park, Cuba. We selected 20 adult plants of each one of the bromeliad species (Tillandsia utriculata, T. fasciculata, Hohenbergia penduliflora). We determined the tree species and height where bromeliads were growing on. Each bromeliad was removed from its tree and the species and number of each amphibian and reptile were listed. The relative abundance and dominance index of each zoological species were calculated and a dominance-diversity graphic was constructed. There were 34 amphibian and reptiles, belonging to eight species (five families). Four species were endemic to Cuba (three amphibians and one reptile). Of all bromeliads sampled $31,67 \%$ had herpetofauna, $67,65 \%$ of all animals were found in T. utriculata. The animals seem to prefer bromeliads 2,31-3,20m above ground (category "Medio"). The dominant species was Eleutherodactylus varians (relative abundance of $55,88 \%$ ). These tank bromeliads can be considered key species, thus their role is paramount in these semideciduous dry forest (mainly $T$. utriculata) and the resources they provide are essential for the herpetofauna, particularly for the endemic anuran E. varians.
\end{abstract}

KEY WORDS: herpetofauna, Tillandsia utriculata, Tillandsia fasciculata, Hohenbergia penduliflora, anurans, Eleutherodactylus

\section{RESUMEN}

Se estudió la herpetofauna asociada a tres especies de bromelias de tanque en un bosque seco semideciduo sobre carso desnudo en el Parque Nacional Guanahacabibes, Cuba. Se seleccionaron 20 plantas adultas de cada una de las especies de bromelias (Tillandsia utriculata, T. fasciculata, Hohenbergia penduliflora). Determinamos las especies y altura de los árboles en los que las bromelias estaban creciendo. Removimos cada bromelia del árbol y se listaron las especies de anfibios y reptiles que se encontraron. Se calculó la abundancia relativa y el índice de dominancia de cada especie zoológica, y construimos un gráfico de diversidad. Había 34 anfibios y reptiles, de ocho especies (cinco familias). Cuatro especies son endémicas de Cuba (tres anfibios y un reptil). De las bromelias muestreadas $31,67 \%$ estaban ocupadas por herpetofauna, $67,65 \%$ de los animales se encontraron en T. utriculata. La herpetofauna tiende a preferir las bromelias a 2,31-3,20m de altura (categoría "Medio"). La especie más dominante fue Eleutherodactylus varians (abundancia relativa de 55,88\%). Estas bromelias de tanque pueden considerarse como especies clave, su papel es primordial en estos bosques secos semideciduos (fundamentalmente T. utriculata) y los recursos que proporcionan son esenciales para la herpetofauna, particularmente para el anuro endémico $E$. varians.

PALABRAS CLAVE: herpetofauna, Tillandsia utriculata, Tillandsia fasciculata, Hohenbergia penduliflora, anuros, Eleutherodactylus.
El dosel de los bosques tropicales es un hábitat prácticamente inexplorado (Basset, Novotny, Miller \& Kitching, 2003), a pesar de estar entre los más ricos de la Tierra (Mitchell et al., 2002; Basset et al., 2003). En él habita aproximadamente $40 \%$ de las especies existentes conocidas y se estima que tiene capacidad para albergar $50 \%$ de la biodiversidad del planeta (Mitchell, Secoy \& Jackson, 2002; Basset et al., 2003). Los anfibios y reptiles en este hábitat han sido muy poco estudiados (De Vries, Murray \& Lande, 1997; Schiesari, Gordo \& Hödl, 2003; Guayasamin, Ron, Cisneros, Lamar \& McCracken, 2006; Cruz-Ruiz, Mondragón \& Santos-Moreno, 2012), principalmente debido a sus hábitos crípticos, lo que dificulta el muestreo (Kays \& Allison, 2001).

De las 65 especies de anfibios que habitan en Cuba (93,8\% de endemismo) (Estrada, 2012), Osteopilus 
septentrionalis (Fig. 1G) y 12 especies de Eleutherodactylus tienen hábitos arborícolas, y presentan diversos grados de adaptación a la vida en los árboles (Rodríguez \& Alonso, 2003). Un grupo particular de ranas arborícolas, principalmente de la familia Eleutherodactylidae, se ha adaptado a la vida en algunas especies de bromelias. Tienen su cuerpo aplanado, apto para la vida en los espacios estrechos que quedan entre las hojas, donde se acumula el agua de lluvia, y sus discos digitales son grandes, lo que les permite trepar por las hojas lisas de estas plantas (Rodríguez \& Alonso, 2003; Díaz \& Cádiz, 2008; Romero et al., 2010). Este tipo de ranas depositan sus huevos y crían sus renacuajos en las propias plantas donde viven, con lo cual cierran su ciclo de vida arborícola sin necesidad de bajar jamás al suelo (Schiesari, Grillitsch \& Vogt, 1996; Campbell, 1998; Rodríguez \& Alonso, 2003; Díaz \& Cádiz, 2008; Romero et al., 2010). De las 156 especies de reptiles (83,3\% de endemismo) (Estrada, 2012), que se encuentran en la isla, varios taxones, incluidos en diferentes familias (ej: Iguanidae, Sphaerodactylidae, Tropidophiidae), frecuentemente también utilizan las bromelias, como refugio o para obtener alimentos (Chamizo, Rodríguez, Moreno, Domiínguez \& Díaz, 2003; Domínguez \& Moreno, 2003).

De los 58 géneros que componen la familia Bromeliaceae (Luther, 2008), aproximadamente 40 tienen fitotelmata, por lo que se les conoce como bromelias de tanque (Fish, 1983). El género Tillandsia es el más diverso de la familia, y las rosetas pueden poseer un tanque formado por las vainas de las hojas en su base (Ramírez, Carnevalli \& Chi, 2004). Como fitotelmata se reconoce a las plantas o partes de ellas que acumulan agua de lluvia y materia orgánica, facilitando el desarrollo de comunidades de organismos (Beutelspacher, 1971; Fish, 1983; Kitching, 2000; Liria, 2007). En particular estas bromelias son capaces de contener cantidades relativamente grandes de agua y desempeñan un papel principal como "recurso clave" en su hábitat, para vertebrados, invertebrados y otras plantas (Nadkarni, 1994; Kitching, 2000; Wittman, 2000; De Carvalho \& De Araújo, 2007; McCracken \& Forstner, 2008; Cruz-Ruiz et al., 2012).

Teniendo en cuenta la gran importancia ecológica de las bromelias de tanque y su fitotelmata dentro de los bosques tropicales (Nadkarni, 1994; McCracken \& Forstner, 2008), en el presente trabajo se estudia la herpetofauna asociada a las tres especies de bromelias de tanque (Tillandsia utriculata L., T. fasciculata Sw. y Hohenbergia penduliflora (A. Rich.) Mez (Fig. 1A, B, C) que habitan en un bosque seco semideciduo, ubicado en la zona de uso público del Parque Nacional Guanahacabibes, Cuba. Se pretende determinar con cuales especies de bromelias están más asociados los anfibios y reptiles en este ecosistema, cuales especies de anfibios y reptiles las utilizan, y las condiciones que deben tener estas plantas para ser buenos hospederos.

\section{MATERIALES Y MÉTODOS}

Caracterización del sitio y de las especies de bromelias: El estudio se desarrolló en 2010, en un área aproximada de 2 ha, en un bosque seco semideciduo sobre carso desnudo (Delgado, 2012), localizado en los $21^{\circ} 55^{\prime} 23,0^{\prime \prime} \mathrm{N}$ y $084^{\circ} 28^{\prime} 38,2^{\prime \prime} \mathrm{W}$, en el área de La Bajada, $300 \mathrm{~m}$ al norte del centro de visitantes del Parque Nacional Guanahacabibes, Pinar del Río, Cuba. El promedio anual de precipitación es de $1431 \mathrm{~mm}$, y las temperaturas promedio de $25,3^{\circ} \mathrm{C}$ (Márquez et al., 2009). En estos bosques crecen simpátricamente diferentes especies de epífitas vasculares, entre las que destacan las bromelias de tanque T. utriculata, T. fasciculata y $H$. penduliflora.

Tillandsia utriculata (Fig. 1A) se distribuye desde Estados Unidos (Florida), hasta Venezuela y las Indias Occidentales (Ramírez et al., 2004). En Cuba se encuentra en todo el país (Hechavarría, 2009). Es fundamentalmente epífita, alcanza hasta $2 \mathrm{~m}$ de alto (con inflorescencia), tiene roseta utriculada, hojas solapadas, con láminas linear-triangulares, de color verde brillante. Inflorescencia central, erecta, ampliamente pinnada, laxa, glabra, escapo igual o excediendo las hojas, brácteas erectas, tubular-involutas, con flores generalmente blancas, cápsulas cilíndricas, agudas, de hasta $4 \mathrm{~cm}$ de largo y semillas con apéndice plumoso apical (Ramírez et al., 2004).

Tillandsia fasciculata (Fig. 1B) habita en México, Mesoamérica, Suramérica y las Indias Occidentales (Ramírez et al., 2004), en Cuba se distribuye por todo el país (Bello \& Torres, 2011; Acevedo-Rodríguez \& Strong, 2012). Es una especie principalmente epífita, de hasta $1 \mathrm{~m}$ de alto (con inflorescencia), con roseta infundibuliforme, hojas café oscuro en la base, con superficie lisa y lustrosa, con láminas estrechamente triangulares. La inflorescencia usualmente es paniculada, con flores violeta-purpúreo, cápsulas rígidas de $4 \mathrm{~cm}$ de largo y semillas de $6 \mathrm{~mm}$ de largo (Smith \& Downs, 1977; Ramírez et al., 2004).

Hohenbergia penduliflora (Fig. 1C) es endémica de las Antillas Mayores (Acevedo-Rodríguez \& Strong, 2012) y se distribuye por toda Cuba (Hechavarría, 2009). Son plantas grandes, epífitas, rupícolas o terrestres, hojas linearliguladas, ampliamente redondeadas con un amplio apículo triangular, de $12 \mathrm{~cm}$ de ancho, presentan bordes aserrados con dientes de $2 \mathrm{~mm}$ de largo. Inflorescencia en panícula erecta, igualando o excediendo en tamaño a las hojas. Brácteas florales anchamente ovadas, acuminadas, de 7-15mm de largo, sépalos fuertemente asimétricos, 

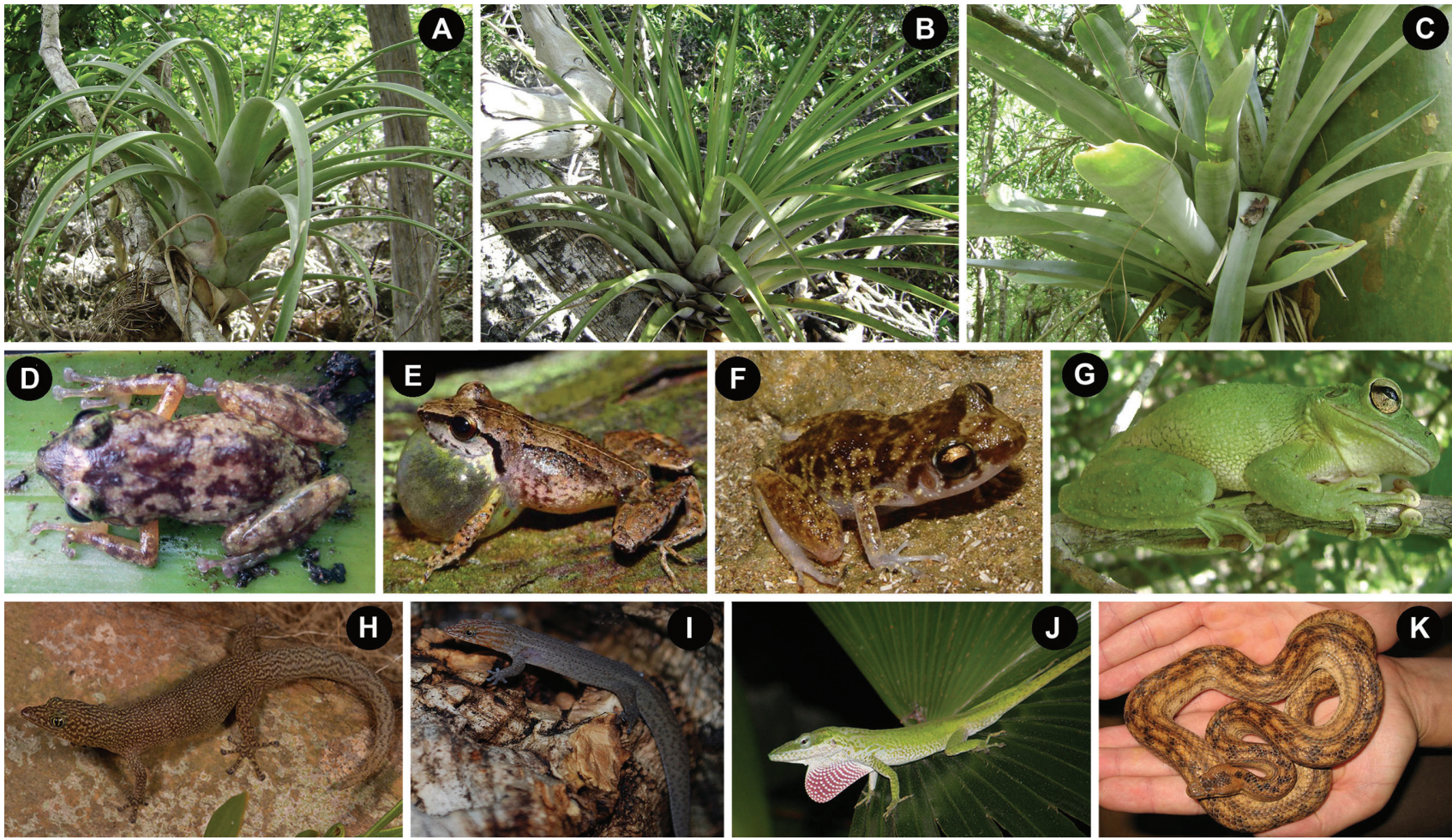

Fig. 1. Especies de bromelias de tanque estudiadas: Tillandsia utriculata (A), T. fasciculata (B) y Hohenbergia penduliflora (C). Especies de anfibios y reptiles que se encontraron ocupando las tres especies de bromelias: Eleutherodactylus varians (D), E. auriculatus (E), E. guanahacabibes $(\mathrm{F})$, Osteopilus septentrionalis $(\mathrm{G})$, Sphaerodactylus elegans elegans $(\mathrm{H})$, S. notatus atactus (I), Anolis porcatus (J), Tropidophis melanurus (K). Las imágenes de cada especie no están a escala entre ellas. (Autores: A, B, C, G- García-González A.; D- García L.Y.; E, F- Rodríguez A.; H- Pierson Hill; I- Yan Fulliquet; J- copépodo; K- peterG).

de 4-8mm de largo, pétalos linear-lanceolados, acuminados, de $12 \mathrm{~mm}$ de largo, blancos, fruto en baya subglobosa (Smith \& Downs, 1979).

Altura y DAP de los árboles: Utilizando la metodología propuesta por García-González et al. (2011), se estimó la altura $(\mathrm{m})$ de cada árbol hospedero de bromelias y se midió su diámetro a la altura del pecho (DAP) $(\mathrm{cm})$. También se estimó la altura en que se ubicaron las bromelias en los árboles y se midió el diámetro de la sección del tronco o de las ramas donde crecían.

Muestreo de anfibios y reptiles: De cada una de las tres especies de bromelias de tanque estudiadas (T. utriculata, $T$. fasciculata y $\mathrm{H}$. penduliflora), se seleccionaron en el campo 20 plantas ( 60 bromelias totales; todas epífitas). Como criterio de selección se tuvo en cuenta que fueran adultas (indicios de emisión del escapo floral), lo que garantizó un tamaño estándar entre los individuos seleccionados de cada especie. En cada caso se identificó la especie de árbol que ocupaban las bromelias.

Cada planta se desprendió cuidadosamente del árbol, tomando en una mano varias hojas por el extremo y cortando con un serrucho su tallo por la base (McCracken \& Forstner, 2008). En lo posible se evitaron los movimientos bruscos y siempre se mantuvo la bromelia en posición vertical, para impedir que escaparan los anfibios y reptiles que la habitaran. La conducta típica de la herpetofauna que ocupa estos microhábitats facilitó que no se perdieran animales, ya que al sentir los primeros movimientos de remoción, generalmente tienden a refugiarse en el fondo de la fitotelmata (McCracken \& Forstner, 2008).

Una vez en el suelo, la planta se colocó dentro de un recipiente plástico circular de $45 \mathrm{~cm}$ de ancho y $20 \mathrm{~cm}$ de alto, que se colocó encima de una manta de nylon blanco de $2 \mathrm{~m} \times 2 \mathrm{~m}$. A cada planta se le fueron desprendiendo cuidadosamente las hojas y anotando las especies y número de cada anfibio o reptil que la ocupaban. El método de muestreo utilizado fue destructivo ya que los anfibios 
y reptiles no pueden ser muestreados sin remover y abrir la bromelia para buscar en cavidades interfoliares (Galindo-Leal, Cedeño-Vásquez, Calderón \& Augustine, 2003; McCracken \& Forstner, 2008). No obstante, estas especies de bromelia tienen una amplia distribución en Cuba (Hechavarría, 2009; Bello \& Torres, 2011; AcevedoRodríguez \& Strong, 2012) y no se encuentran bajo ninguna categoría de amenaza en el país o en la provincia (Berazaín, Areces, Lazcano \& González-Torres, 2005; Urquiola, González-Oliva, Novo \& Acosta, 2010), por lo que su conservación no se ve comprometida por la realización de este estudio.

Para calcular la abundancia relativa de la especie zoológica más abundante se empleó la fórmula propuesta por Ramírez-Villarroel (1993). Adicionalmente se calculó el índice de dominancia usando la fórmula propuesta por McNaughton (1968). Con el fin de conocer y comparar los valores de abundancia de todas las especies de anfibios y reptiles encontrados en las tres especies de bromelias, se construyó un gráfico de dominancia-diversidad (Feinsinger, 2003).

\section{Identificación de los árboles y de la herpetofauna:} Para la identificación taxonómica de los árboles donde crecían las bromelias, y de los anfibios y reptiles encontrados, se tuvo en cuenta la experiencia en campo de los autores, apoyándose en Bisse (1988), Acevedo-Rodríguez \& Strong (2012), Rodríguez (1999), Díaz \& Cádiz (2008), Estrada (2012) y Hedges (2013).

Análisis estadísticos: Utilizando el programa StatXact-4 (Versión 4.0.1), se hizo una comparación entre las frecuencias de aparición por medio de una prueba de Montecarlo. Este algoritmo se empleó en el caso de las variables: alturas a las que crecen las tres especies de bromelias y número de anfibios y reptiles que utilizan cada especie de bromelia. Se utilizaron los programas PopTools para hacer una prueba de correlación de Mantel (con 10000 permutaciones), y el SPSS 15.0, para hacer un gráfico de frecuencias con las categorías de altura a las que se encontraron las bromelias con presencia de anfibios y reptiles. Los datos de altura de todas las bromelias muestreadas se dividieron equitativamente en tres categorías (Bajo: 0,4-2,3m, Medio: 2,31-3,2m, Alto: 3,21-6m), teniendo en cuenta los valores mínimo y máximo de altura. Se aplicaron como estadísticos descriptivos la media y la desviación estándar.

\section{RESULTADOS}

Bromelias recolectadas, altura, diámetro y especies de árboles sobre los que crecían: Las tres especies de bromelias estudiadas se recolectaron en 15

CUADRO 1

Especies de árboles donde crecían las tres especies de bromelias de tanque estudiadas (Tillandsia utriculata, T. fasciculata, Hohenbergia penduliflora), familia botánica a la que pertenecen, número y composición de las bromelias muestreadas en cada especie de árbol.

\begin{tabular}{|c|c|c|c|c|c|}
\hline \multirow{2}{*}{ Especies de árboles } & \multirow{2}{*}{ Familia } & \multirow{2}{*}{$\begin{array}{l}\text { No. de } \\
\text { bromelias }\end{array}$} & \multicolumn{3}{|c|}{ No. de bromelias en cada árbol } \\
\hline & & & T. utriculata & T. fasciculata & H. penduliflora \\
\hline Adelia ricinella $\mathrm{L}$. & Euphorbiaceae & 2 & 1 & 1 & 0 \\
\hline Bombacopsis cubensis A. Robyns & Bombacaceae & 4 & 0 & 1 & 3 \\
\hline Capparis cynophallophora L & Capparaceae & 1 & 0 & 1 & 0 \\
\hline Capparis flexuosa (L.) L. & Capparaceae & 3 & 0 & 2 & 1 \\
\hline Cedrela odorata L. & Meliaceae & 1 & 0 & 0 & 1 \\
\hline Celtis trinervia Lam. & Cannabaceae & 1 & 1 & 0 & 0 \\
\hline Citharexylum fruticosum L. & Verbenaceae & 1 & 0 & 1 & 0 \\
\hline Erythroxylum areolatum L. & Erythroxylaceae & 3 & 1 & 2 & 0 \\
\hline Ficus aurea Nutt. & Moraceae & 6 & 0 & 0 & 6 \\
\hline Ficus maxima Mill. & Moraceae & 1 & 1 & 0 & 0 \\
\hline Ficus trigonata $\mathrm{L}$. & Moraceae & 1 & 1 & 0 & 0 \\
\hline Gerascanthus gerascanthoides (Kunth) Borhidi & Boraginaceae & 16 & 10 & 6 & 0 \\
\hline Plumeria tuberculata Lodd & Apocynaceae & 7 & 4 & 2 & 1 \\
\hline Árbol seco & - & 11 & 1 & 2 & 8 \\
\hline Tabebuia myrtifolia (Gris.) Britt & Bignoniaceae & 1 & 0 & 1 & 0 \\
\hline Trichilia hirta L. & Meliaceae & 1 & 0 & 1 & 0 \\
\hline Total & - & 60 & 20 & 20 & 20 \\
\hline
\end{tabular}


especies de árboles, pertenecientes a 11 familias botánicas (Cuadro 1). Uno de los árboles no fue posible identificarlo por estar seco (Cuadro 1). La altura promedio de los árboles fue $7,05 \pm 3,12 \mathrm{~m}$ y el DAP promedio $19,11 \pm 15,77 \mathrm{~cm}$. La altura promedio a la que crecían las tres especies de bromelias fue $2,86 \pm 1,18 \mathrm{~m}$ y el DAP promedio $15,61 \pm 14,73 \mathrm{~cm}$. No existen diferencias significativas entre las alturas a las que se encontraron las tres especies de bromelias (Montecarlo, $p=0,069$ ).

Anfibios y reptiles encontrados: Teniendo en cuenta las tres especies de bromelias estudiadas, se encontraron 34 anfibios y reptiles, 30 de ellos adultos y cuatro juveniles (dos Anolis porcatus (Fig. 1J), un O. septentrionalis (Fig. 1G) y un Sphaerodactylus elegans elegans (Fig. 1H)), de ocho especies (cuatro de anfibios (anuros) y cuatro de reptiles), incluidas en cinco familias zoológicas (Cuadro 2.). Son endémicas de Cuba cuatro de las especies localizadas (50\%) (Cuadro 2.).

\section{Ocupación de las bromelias y correlación de este parámetro con la altura a la que crecían las plantas:} De las bromelias muestreadas 31,67\% (19 bromelias) estaban ocupadas por anfibios y reptiles. En T. utriculata se encontró $67,65 \%$ de los animales, en $H$. penduliflora $23,53 \%$ y en $T$. fasciculata $8,82 \%$, existiendo diferencias significativas entre el número de anfibios y reptiles que utilizan cada especie de bromelia (Montecarlo, $p=0,0089$ ).

Se encontraron anfibios y reptiles en bromelias hasta $6 \mathrm{~m}$ de altura, pero se aprecia una tendencia de la herpetofauna a preferir las bromelias que crecen a alturas entre 2,31 y 3,2m (categoría "Medio" de altura) (Fig. 2). De las bromelias ocupadas $42,1 \%$ crecieron en este rango de altura. Con los datos utilizados no hay suficientes elementos para asegurar que el número de animales que ocupan las bromelias esté relacionado con la altura a la que éstas se encuentran en el árbol, ya que valores del índice de correlación de Mantel superiores al observado se pueden obtener por azar con una frecuencia de $31 \%$.

Abundancia relativa y dominancia de las especies de anfibios y reptiles: y De las especies de anfibios y reptiles encontrados, la especie dominante fue Eleutherodactylus varians (Fig. 3; Fig. 1D), con una abundancia relativa de 55,88\%. La dominancia general es de $64,7 \%$, determinada fundamentalmente por $E$. varians.

\section{CUADRO 2}

Especies de anfibios y reptiles encontrados ocupando las tres especies de bromelias de tanque estudiadas, familia zoológica a la que pertenecen, estatus y número de individuos por especie de bromelia (Tillandsia utriculata, T. fasciculata, Hohenbergia penduliflora).

\begin{tabular}{|c|c|c|c|c|c|}
\hline \multirow{2}{*}{ Anfibios y reptiles } & \multirow{2}{*}{ Familia } & \multirow{2}{*}{ Estatus* } & \multicolumn{3}{|c|}{ No. de animales por especie de bromelia } \\
\hline & & & T. utriculata & T. fasciculata & H. penduliflora \\
\hline \multicolumn{6}{|c|}{ Anfibios } \\
\hline Eleutherodactylus auriculatus Cope & Eleutherodactylidae & En & 2 & 1 & 0 \\
\hline $\begin{array}{l}\text { Eleutherodactylus guanahacabibes } \\
\text { Estrada \& Rodriguez }\end{array}$ & Eleutherodactylidae & En & 2 & 0 & 1 \\
\hline $\begin{array}{l}\text { Eleutherodactylus varians Gundlach \& } \\
\text { Peters }\end{array}$ & Eleutherodactylidae & En & 17 & 0 & 1 \\
\hline $\begin{array}{l}\text { Osteopilus septentrionalis Duméril \& } \\
\text { Bibron }\end{array}$ & Hylidae & $\mathrm{Au}$ & 0 & 1 & 0 \\
\hline Total & - & - & 21 & 2 & 2 \\
\hline \multicolumn{6}{|c|}{ Reptiles } \\
\hline Anolis porcatus Gray & Iguanidae & $\mathrm{Au}$ & 0 & 0 & 3 \\
\hline $\begin{array}{l}\text { Sphaerodactylus elegans elegans } \\
\text { Macleay }\end{array}$ & Sphaerodactylidae & $\mathrm{Au}$ & 1 & 0 & 1 \\
\hline $\begin{array}{l}\text { Sphaerodactylus notatus atactus } \\
\text { Schwartz }\end{array}$ & Sphaerodactylidae & $\mathrm{Au}$ & 1 & 0 & 2 \\
\hline Tropidophis melanurus Schlegel & Tropidophiidae & En & 0 & 1 & 0 \\
\hline Total & - & - & 2 & 1 & 6 \\
\hline Total general & - & - & 23 & 3 & 8 \\
\hline
\end{tabular}

*En: endémica, Au: autóctona. 


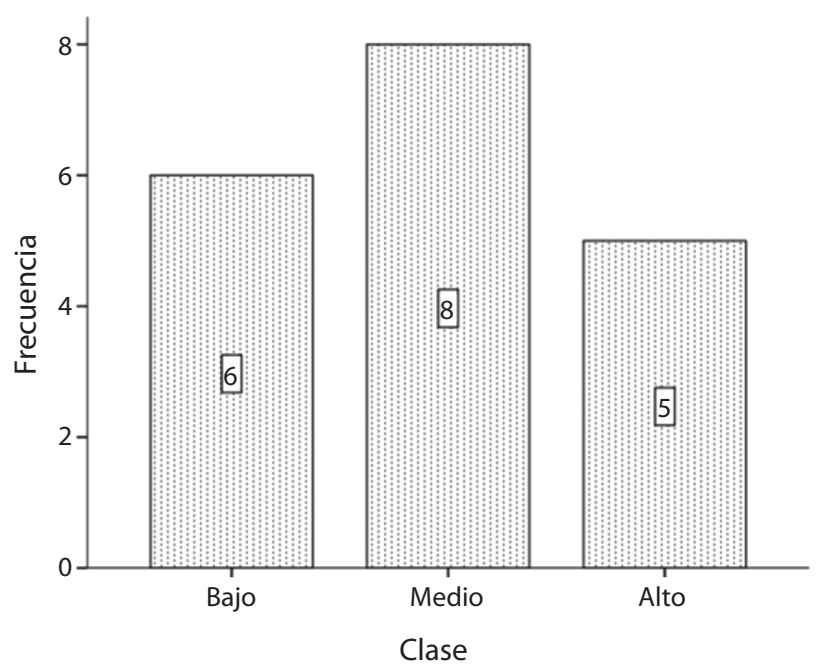

Fig. 2. Categorías de altura $(m)$ en las que crecían en los árboles las tres especies de bromelias de tanque estudiadas (Tillandsia utriculata, T. fasciculata, Hohenbergia penduliflora) y número de bromelias ocupadas por anfibios y reptiles en cada categoría. Categorías de altura: Bajo: 0,4-2,3m, Medio: 2,31-3,2m, Alto: 3,21-6m.

En T. utriculata es de $86,95 \%$, determinada por E. varians, en $H$. penduliflora es $62,5 \%$ determinada por Anolis porcatus y en $T$. fasciculata las tres especies de anfibios y reptiles presentaron solo un individuo cada una (Cuadro 2). En las colas de las curvas se observan siete especies, tres en $T$. fasciculata, tres en $H$. penduliflora y una en T. utriculata (Fig. 3).

\section{DISCUSIÓN}

En cuanto a número de especies encontradas predominó el género Eleutherodactylus (Cuadro 2) y hubo un alto índice de endemismo, lo cual es típico de la herpetofauna cubana (Fong, 2000; Rodríguez, 2003; Díaz \& Cádiz, 2008; Estrada, 2012).

Muchas bromelias proporcionan condiciones ideales como, temperatura estable, humedad, alimento y/o protección contra depredadores (Beutelspacher, 1971; Benzing, 1980; Benzing, 1990; Dejean, Olmsted \& Snelling, 1995; Galindo-Leal et al., 2003; De Carvalho \& De Araújo, 2007; McCracken \& Forstner, 2008; MondragónChaparro \& Cruz-Ruiz, 2009; Cruz-Ruiz et al., 2012). En las diferentes especies de bromelias, la disponibilidad de fitotelmata y de los recursos que proporciona este microhábitat, puede jugar un importante papel en la presencia-ausencia de una gran variedad de organismos (Picado, 1913; Nadkarni, 1994; Richardson, 1999; Benzing, 2000; Wittman, 2000; Galindo-Leal et al., 2003; Liria, 2007; McCracken \& Forstner, 2008; Frank \& Lounibos, 2009). Sólo de agua, uno de los principales recursos que proporciona la fitotelmata, las reservas para los organismos asociados puede llegar a 50 000L/ha en algunos lugares tropicales (Kitching, 2000; McCracken \& Forstner, 2006). Algunos organismos muestran una marcada especificidad de ocupación hacia algunas especies de bromelias (Romero, 2006), aunque la mayoría son principalmente generalistas (Richardson, Rogers \& Richardson, 2000; Stuntz, 2001).

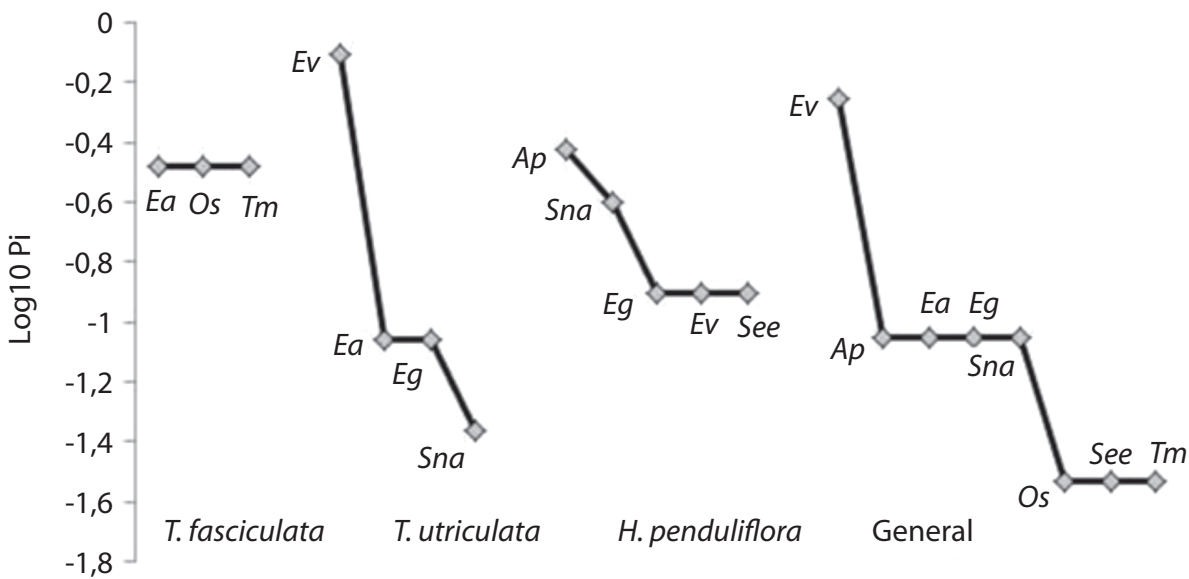

Fig. 3. Dominancia-diversidad de especies de anfibios y reptiles en las tres especies de bromelias de tanque estudiadas (Tillandsia fasciculata, T. utriculata, Hohenbergia penduliflora). Especies de anfibios y reptiles: (Ap) Anolis porcatus, (Ea) Eleutherodactylus auriculatus, (Eg) E. guanahacabibes, (Ev) E. varians, (Os) Osteopilus septentrionalis, (See) Sphaerodactylus elegans elegans, (Sna) S. notatus atactus, (Tm) Tropidophis melanurus. 
Las tres especies de bromelias estudiadas pueden considerarse como bromelias de tanque, pero difieren en su tamaño y morfología, y por tanto en su disponibilidad de fitotelmata (Smith \& Downs, 1977; 1979; Ramírez et al., 2004). Este microhábitat es escaso en T. fasciculata, intermedio en T. utriculata y máximo en $H$. penduliflora, lo que probablemente tiene gran influencia en los diferentes niveles de ocupación por anfibios y reptiles (Cuadro 2). El menor número de animales se localizó en T. fasciculata, y los que la ocupaban no son estrictamente bromelícolas (Cuadro 2). McCracken \& Forstner (2008), no encontraron ni anfibios ni reptiles ocupando tres plantas de una especie de bromelia sin tanque no identificada, que muestrearon en un bosque siempreverde bajo amazónico en Ecuador. No obstante, muchas bromelias que debido a la morfología de la planta, la edad o ubicación física, no tienen fitotelmata o ésta es escasa, también pueden ser utilizadas por varios animales (Wittman, 2000).

De la herpetofauna encontrada en $T$. fasciculata (Cuadro 2), Eleutherodactylus auriculatus (Fig. 1E) es una especie abundante en muchas áreas boscosas de Cuba y frecuentemente utiliza las bromelias, pero es generalista para varios nichos dentro del bosque, adaptándose a una amplia variedad de microhábitats (Díaz \& Cádiz, 2008; IUCN, 2013a).

Osteopilus septentrionalis es una especie arborícola, de amplia distribución en el país, se adapta a un elevado nivel de disturbio y es considerada invasora en varias regiones del neotrópico (Rivalta \& Díaz, 2003; Rodríguez \& Alonso, 2003; Díaz \& Cádiz, 2008; IUCN, 2013b). Teniendo en cuenta estas características, probablemente utilice la bromelia como refugio diurno (Galindo-Leal et al., 2003; Rivalta \& Díaz, 2003; Romero et al., 2010), pero su presencia en $T$. fasciculata no debe responder a ninguna característica ecológica en particular.

Tropidophis melanurus (Fig. 1K) frecuentemente se localiza ocupando bromelias, las que utiliza como refugio o para cazar insectos y pequeños vertebrados de los que se alimenta, especialmente ranas del género Eleutherodactylus, que comúnmente habitan en las bromelias (Domínguez \& Moreno, 2003).

De las tres especies de bromelias, $H$. penduliflora es la de mayor tamaño y con mayor espacio entre las vainas foliares, siendo capaz de acumular un gran volumen de agua en las axilas de las hojas, por lo que de las tres especies estudiadas se le puede considerar la mejor representante entre las bromelia de tanque en el área de estudio. McCracken \& Forstner (2008), muestrearon 57 bromelias de tanque de las especies Aechmea zebrina y Aechmea sp., especies muy similares a $H$. penduliflora, encontrando cuatro especies de anuros, incluyendo dos especies de
Eleutherodactylus. También Galindo-Leal et al. (2003), en Campeche, México, localizó tres especies de ranas arborícolas y una salamandra, en una muestra de 60 bromelias de tanque de la especie Aechmea bracteata. Se encontraron anuros en $65 \%$ de las muestras de A. zebrina ( 26 plantas), en $29,41 \%$ de las de Aechmea sp. (cinco plantas) (McCracken \& Forstner, 2008) y en $27 \%$ de las de A. bracteata (16 plantas) (Galindo-Leal et al., 2003), en contraste con $10 \%$ (dos plantas) en el caso de $H$. penduliflora en nuestro estudio, lo que posiblemente esté relacionado con la morfología de esta planta y la morfología y conducta de los animales que se encontraron ocupándola.

De los anfibios y reptiles que se encontraron en $H$. penduliflora (Cuadro 2), A. porcatus es un reptil muy adaptable de amplia distribución en todo el país (Rodríguez, 1999) y probablemente prefiera la especie de bromelia de tanque más grande al brindarle mayores posibilidades de obtener alimentos, por acumular mayor cantidad de agua y materia orgánica, y posiblemente tener una concurrencia de artrópodos más rica y abundante (Beutelspacher, 1971; Benzing, 1990; Dejean et al., 1995; Wittman, 2000; Frank \& Lounibos, 2009). Probablemente ocurra lo mismo con S. elegans elegans y Sphaerodactylus notatus atactus (Fig. 1I) (Chamizo et al., 2003), pudiendo encontrarlos también en T. utriculata, que es la segunda especie de bromelia con mayor disponibilidad de fitotelmata. En Cuba, S. elegans elegans es muy abundante y generalista, mientras que $S$. notatus atactus solo se localiza en diferentes microhábitats dentro de zonas boscosas (Chamizo et al., 2003). En Baracoa, Cuba, Sphaerodactylus bromeliarum habita exclusivamente en bromelias (Chamizo et al., 2003) y en diferentes regiones neotropicales otros lagartos como Abronia oaxacae, Bolitoglossa rufescens, $B$. mulleri, B. mexicana, B. subpalmata, B. yucatana, Hemidactylus mabouia, Gymnodactylus darwinii, Mabuya agilis y M. macrorhyncha, también han sido localizados utilizando bromelias de tanque como refugio (Scott \& Limerick, 1983; Lee, 1996; Campbell, 1998; Galindo-Leal et al., 2003; De Carvalho \& De Araújo, 2007; Cruz-Ruiz et al., 2012).

Eleutherodactylus guanahacabibes (Fig. 1F) es una especie terrestre, que vive fundamentalmente en los ambientes rocosos calizos que predominan en la Península de Guanahacabibes, de donde es endémica local (Alonso \& Rodríguez, 2003; Díaz \& Cádiz, 2008; IUCN, 2013c), y en la temporada lluviosa es frecuente que se disperse por todo el hábitat pétreo de la Península (Alonso \& Rodríguez, 2003). No obstante, probablemente su presencia en bromelias sea casual, las utilice como refugio y para buscar pequeños invertebrados de los cuales se alimenta. Los tres individuos de E. guanahacabibes que se encontraron ocupando este microhábitat, siempre se 
localizaron en bromelias por debajo de los $3 \mathrm{~m}$ de altura. Eleutherodactylus varians es uno de los anuros cubanos con mayor especialización para la vida en las bromelias, siendo posible encontrarlo desde la Península de Guanahacabibes, al occidente de Cuba, hasta la Sierra de Cubitas, en la porción centro-oriental del país, en bosques bien conservados (Rodríguez \& Alonso, 2003; Díaz \& Cádiz, 2008), como los del área donde se desarrolló el estudio.

Tillandsia utriculata es la segunda especie de bromelia con mayor disponibilidad de fitotelmata (más que $T$. fasciculata y menos que $H$. penduliflora), localizándose en ella a las dos especies de Sphaerodactylus encontradas y a las tres de Eleutherodactylus (Cuadro 2). Probablemente el microhábitat intermedio que proporciona esta bromelia le brinda mejores condiciones a algunas especies de animales, fundamentalmente a $E$. varians. Es posible que el espacio entre las brácteas de $T$. utriculata sea el más adecuado para darle protección y que este anuro bromelícola desarrolle su ciclo de vida, siendo más amplio que el espacio de T. fasciculata, pero no tan ancho y con tanta acumulación de agua como el de $H$. penduliflora.

De forma general en cuanto a la dominancia de especies, E. varians fue la especie más abundante, con un ámbito amplio de diferencia respecto a las demás (Cuadro 2, Fig. 3). Las especies de anfibios y reptiles en las colas de las curvas (Fig. 3) pueden ser consecuencia de un azar del muestreo, pero es más factible que sean consecuencia de las características propias de cada una de las especies de bromelias estudiadas y las posibilidades de refugio, alimentación y reproducción que les brindan a la herpetofauna.

A pesar de que algunos autores señalan que la abundancia, composición y distribución de las epífitas pueden estar determinadas por la especie de árbol, sus características físicas (ej: edad, tamaño y tipo de corteza) y por condiciones microclimáticas (ej: intensidad luminosa, humedad y corrientes de aire) (Benzing, 1980; Shaw, 2005; Bonnet \& Queiroz, 2006; Kersten \& Silva, 2006; García-Franco \& Toledo, 2008), las bromelias muestreadas se encontraron y recolectaron a varias alturas (Fig. 2), en varias especies de árboles, y aparentemente la especie de árbol no fue determinante en el número de bromelia que los ocupaban (Cuadro 1). No obstante, se observó una preferencia de las bromelias a crecer en la porción intermedia de los árboles, donde probablemente también sean intermedias la intensidad luminosa, la humedad y la intensidad de las corrientes de aire. También se aprecia una tendencia de T. utriculata y T. fasciculata a ocupar mayoritariamente Gerascanthus gerascanthoides (16 bromelias sobre esta especie) (Cuadro 1), lo cual posiblemente se relacione con que esta especie de árbol sea uno de los componentes principales de los bosques semideciduos en la Península de Guanahacabibes, siendo muy abundante en todos sus estadios sucesionales (Delgado, 2012). En el caso de H. penduliflora, no se encontró ningún individuo ocupando este árbol (Cuadro 1), lo cual probablemente sea consecuencia de que esta especie de bromelia alcanza gran tamaño, por lo que requiere árboles de fuste y ramas gruesas, que sean capaces de sostener su peso. Generalmente en estos bosques las especies arbóreas con una alta densidad, como G. gerascanthoides, coinciden con las de menores diámetros y alturas, aunque tienen un comportamiento diferente en otros tipos de bosques fuera de la región (Delgado, 2012).

A pesar de que con los datos utilizados no queda clara la relación entre el número de animales que ocupan las bromelias y la altura a la que éstas se encuentran en los árboles, es muy probable que estas variables si estén directamente relacionadas. Es importante señalar que el árbol, las condiciones físicas del sitio donde crece la bromelia y los recursos circundantes a la bromelia, influyen en la estructura y composición de la fitotelmata (Palacios-Vargas, 1979).

La preferencia de la herpetofauna por las bromelias que crecen entre 2,31 y $3,2 \mathrm{~m}$ de altura (Fig. 2), puede ser consecuencia de las características propias del bosque seco semideciduo sobre carso desnudo donde se desarrolló el estudio. Este tipo de bosque estructuralmente tiene un solo estrato arbóreo de 8 a $12 \mathrm{~m}$ de altura generalmente, sin sobrepasar nunca los $16 \mathrm{~m}$, y un estrato arbustivo poco denso de $1 \mathrm{a} 4 \mathrm{~m}$ de altura. El dosel es abierto, con copas anchas y más del 50\% de los árboles que lo componen son de especies que en la temporada seca pierden todas o parte de sus hojas (Delgado, 2012). Bajo estas condiciones, las bromelias que crecen en el dosel medio son las que le brindan mejores condiciones y mayor protección a los anfibios y reptiles que las ocupan, particularmente a los anuros bromelícolas. De forma similar, en el estudio desarrollado por Galindo-Leal et al. (2003), el mayor número de anuros se localizó en bromelias que se encontraban de los 2,1 a los $4 \mathrm{~m}$ de altura.

Probablemente los anfibios y reptiles que ocupan las bromelias que crecen a muy baja altura están más expuestos a los depredadores terrestres. También estas bromelias tienen menos exposición a la lluvia y a la caída de hojas y otros productos vegetales del dosel superior, por lo que posiblemente su fitotelmata sea más pobre. Por otra parte, las bromelias del dosel superior reciben más directamente el agua de lluvia y los productos de desecho de los árboles, pero la fauna que las ocupa está más expuesta a depredadores aéreos y a factores 
ambientales extremos. Por ejemplo, los fuertes vientos pueden influir en la mayor pérdida de plantas por daños mecánicos en el dosel, y el sol más directo, en la desecación más rápida de su fitotelmata, fundamentalmente en la temporada poco lluviosa.

Teniendo en cuenta que las bromelias pueden ser refugios especialmente importantes para los anfibios en los bosques estacionales, donde los tanques son uno de los pocos lugares que conservan agua durante los meses poco lluviosos (Galindo-Leal et al., 2003). Probablemente en los bosques secos semideciduos, donde se realizó el estudio, esta dependencia entre la herpetofauna y la fitotelmata se sobredimensiona durante estos meses, dada la gran infiltración que caracteriza los suelos de la Península de Guanahacabibes, haciendo muy escasas las fuentes de agua superficiales, particularmente en la estación seca (Márquez et al., 2009).

Los anfibios se pueden considerar un homólogo vertebrado de las epífitas como especies bioindicadoras, y su utilización del microhábitat de las bromelias de tanque posibilita evaluar el grado de perturbación en el dosel de los bosques (McCracken \& Forstner, 2006). Esto nos permite afirmar que las especies de bromelias muestreadas, de acuerdo con su elevado nivel de ocupación por anfibios y reptiles, pueden considerarse como especies claves (Galindo-Leal et al., 2003). Su papel es primordial en el ecosistema que ocupan (principalmente T. utricula$t a)$, y los recursos que proporcionan son fundamentales para la herpetofauna, particularmente para el anuro endémico E. varians.

\section{AGRADECIMIENTOS}

A Dennis Denis Ávila por sus invaluables conocimientos y su tiempo, puestos en función de este estudio. A los trabajadores del Parque Nacional Guanahacabibes, especialmente a José Alberto Camejo y a Dorka Cobián Rojas, por el apoyo brindado en la realización de este trabajo. A Jorge Ferro Días por las valiosas ideas aportadas. A la organización IDEA WILD por el equipamiento proporcionado, fundamental para la realización de este trabajo. A los revisores anónimos, por sus oportunas correcciones y recomendaciones.

\section{REFERENCIAS}

Acevedo-Rodríguez, P., \& Strong, M. T. (2012). Catalogue of seed plants of the West Indies. Smithsonian Contributions to Botany. Número 98. Washington D.C., E.E.U.U.: Smithsonian Institution Scholarly Press.
Alonso, R., \& Rodríguez, A. (2003). Insospechados habitantes de las penumbras. En L. Rodríguez (Ed.), Anfibios y Reptiles de Cuba (pp. 20-29). Instituto de Ecología y Sistemática (IES), Cuba. Vaasa, Finlandia: UPC Print.

Basset, Y., Novotny, V., Miller, S. E., \& Kitching, R. L. (Eds.). (2003). Arthropods of tropical forests: spatio-temporal dynamics and resource use in the canopy. Cambridge, Reino Unido: Cambridge University Press.

Bello, O. C., \& Torres, Y. (2011). Monopelopia tillandsia (Diptera: Chironomidae: Tanypodinae), primer registro para Cuba y el Neotrópico. Revista Colombiana de Entomología, 37, 162-163.

Benzing, D. H. (1980). The biology of the bromeliads. Eureka, California, E.E.U.U.: Mad River Press Inc.

Benzing, D. H. (1990). Vascular Epiphytes. General biology and related biota. Cambridge, Reino Unido: Cambridge University Press.

Benzing, D. H. (2000). Bromeliaceae: Profile of an Adaptive Radiation. Cambridge, Reino Unido: Cambridge University Press.

Berazaín, R., Areces, F., Lazcano, J. C., \& González-Torres, L. R. (2005). Lista roja de la flora vascular cubana. Documentos del Jardín Botánico Atlántico (Gijón), 4, 1-86.

Beutelspacher, C. R. (1971). Una bromeliácea como ecosistema. Instituto de Biología, Universidad Nacional Autónoma de México (UNAM). Biología, 2, 82-87.

Bisse, J. (1988). Árboles de Cuba. La Habana, Cuba: Científico Técnica.

Bonnet, A., \& Queiroz, M. H. (2006). Estratificação vertical de bromelias epifíticas em diferentes estádios sucessionais da Floresta Ombrófila Densa, Santa Catarina, Brasil. Revista Brasileira de Botânica, 29, 217-228.

Campbell, J. A. (1998). Amphibians and reptiles of northern Guatemala, the Yucatan, and Belize. Oklahoma, E.E.U.U: University of Oklahoma Press.

Chamizo, A., Rodríguez, L., Moreno, L. V., Domínguez, M., \& Días, L. M. (2003). Gigantes y enanos (pp. 74-89). En L. Rodríguez (ed.). Anfibios y Reptiles de Cuba. Instituto de Ecología y Sistemática (IES), Cuba. Vaasa, Finlandia: UPC Print.

Cruz-Ruiz, G. I., Mondragón, D., \& Santos-Moreno, A. (2012). The presence of Abronia oaxacae (Squamata: Anguidae) in tank bromeliads in temperate forests of Oaxaca, Mexico. Brazilian Journal of Biology, 72, 337-341.

DeCarvalho, A.L.G., \&De Araújo, A.F.B. (2007).Ecomorphometric structure of Restinga da Marambaia lizard community, Rio de Janeiro, southeastern Brazil. Revista Brasileira de Zoología, 24, 786-792.

Dejean, A., Olmsted, J., \& Snelling, R. R. (1995). Tree-epiphyteant relationships in the low inundated forest of Sian Ka'an Reserve, Quintana Roo, Mexico. Biotropica, 27, 57-70. 
Delgado, F. (2012). Clasificación funcional del bosque semideciduo de la Reserva de la Biosfera Península de Guanahacabibes, Cuba. (tesis doctoral). Universidad de Alicante, España.

De Vries, P. J., Murray, D., \& Lande, R. (1997). Species diversity in vertical, horizontal, and temporal dimensions of a fruit-feeding butterfly community in an Ecuadorian rainforest. Biological Journal of the Linnean Society, 62, 343-364.

Díaz, L. M., \& Cádiz, A. 2008. Guía taxonómica de los anfibios de Cuba. Fig.3 (Regiones climáticas de Cuba). Volumen 4. Abc Taxa. Bélgica: Belgian Development Cooperation.

Domínguez, M., \& Moreno, L. V. (2003). Serpientes del suelo. En L. Rodríguez (Ed.). Anfibios y Reptiles de Cuba (pp. 98109). Instituto de Ecología y Sistemática (IES), Cuba. UPC Print, Vaasa, Finlandia.

Estrada, A. R. (2012). The Cuban archipelago. En Powell, R., \& Henderson, R. W. (Eds.). Island lists of West Indian amphibians and reptiles. Bulletin of the Florida Museum of Natural History, 51, 85-166.

Feinsinger, P. (2003). El diseño de estudios de campo para la conservación de la biodiversidad. Santa Cruz de la Sierra, Santa Cruz, Bolivia: FAN.

Fish, D. (1983). Phytotelmata: flora and fauna. En Frank J. H., \& Lounibos L. P. (Eds.). Phytotelmata: terrestrial plants as hosts of aquatic insect communities (pp. 161-190). Medford, E.E.U.U: Plexus.

Fong, A. (2000). Anfibios y reptiles del macizo montañoso Sierra Maestra, Cuba: composición, distribución y aspectos ecológicos. Biodiversidad de Cuba Oriental, 5, 124-132.

Frank, J. H., \& Lounibos, L. P. (2009). Insects and allies associated with bromeliads: a review. Terrestrial Arthropod Reviews, $1,125-153$

Galindo-Leal, C., Cedeño-Vázquez, J. R., Calderón, R., \& Augustine, J. (2003). Arboreal frogs, tank bromeliads and disturbed seasonal tropical forest. Contemporary Herpetology, 1, 1-14.

García-Franco, G., \& Toledo, T. (2008). Epífitas vasculares: Bromelias y Orquídeas. En Manson, R. H., Hernandez-Ortíz, V., Gallina, S., \& Mehltreter, K. (Eds.). Agroecosistemas cafetaleros de Veracruz: biodiversidad, manejo y conservación (pp. 69-82). México D.F., México: Instituto de Ecología A.C. (INECOL) e Instituto Nacional de Ecología (INE-SEMARNAT).

García-González, A., Damon, A., Esparza, L. G., \& Valle-Mora, J. (2011). Population structure of Oncidium poikilostalix (Orchidaceae), in coffee plantations in Soconusco, Chiapas, México. Lankesteriana, 11, 23-32.

Guayasamin, J. M., Ron, S., Cisneros-Heredia, D. F., Lamar, W., \& McCracken, S. F. (2006). A new species of frog of the Eleutherodactylus lacrimosus assemblage (Leptodactylidae) from the western Amazon Basin, with comments on the utility of canopy surveys in lowlands rainforest. Herpetologica, 62, 191-202.
Hechavarría, L. (2009). Bromeliaceae. In L.R. GonzálezTorres, R. Rankin, A.T. Leiva, D. Barrios \& A. Palmarola (Eds.).Categorización preliminar de taxones de la flora de Cuba. Bissea (número especial), 3, 50-54.

Hedges, S. B. (2013). Caribherp. West Indian Amphibians and Reptiles. Cuba. Recuperado de http://www.caribherp. org/

IUCN (International Union for Conservation of Nature), (2013a). Eleutherodactylus auriculatus. IUCN Red List of Threatened Species. Version 2013.1. Recuperado de http://www.iucnredlist.org/details/56441/0

IUCN (International Union for Conservation of Nature), (2013b). Osteopilus septentrionalis. IUCN Red List of Threatened Species. Version 2013.1. Recuperado de http://www.iucnredlist.org/details/55811/0

IUCN (International Union for Conservation of Nature), (2013c). Eleutherodactylus guanahacabibes. IUCN Red List of Threatened Species. Version 2013.1. Recuperado de http://www.iucnredlist.org/details/56636/0

Kays, R., \& Allison, A. (2001). Arboreal tropical forest vertebrates: current knowledge and research trends. Plant Ecology, $153,109-120$.

Kersten, A. R., \& Silva, M. S. (2006). The floristic compositions of vascular epiphytes of a seasonally inundated forest on the coastal plain of Ilha do Mel Island, Brazil. Revista de Biología Tropical, 54, 935-942.

Kitching, R. L. (2000). Food webs and container habitats. The natural history and ecology of phytotelmata. Cambridge, Reino Unido: Cambridge University Press.

Lee, J. C. (1996). The amphibians and reptiles of the Yucatan Peninsula. Ithaca, Nueva York, E.E.U.U.: Cornell University Press.

Liria, J. (2007). Fauna fitotelmata en las bromelias Aechmea fendleri André y Hohenbergia. Revista Peruana de Biología (número especial), 14, 033-038.

Luther, H. E. (2008). An alphabetical list of bromeliad binomials (11 th ed.). The Marie Selby Botanical Gardens. Sarasota, Florida, E.E.U.U.: Bromeliad Society International.

Márquez, L., Borrego, O., Camejo, J. A., Cobián, D., Linares, J. L., Rojas, A., Sosa, A., \& Puente, M. (Eds.) (2009). Plan de Manejo Parque Nacional Guanahacabibes (2009-2013). Cuba: Centro de Investigaciones y Servicios Ambientales (ECOVIDA).

McCracken, S. F., \& Forstner, M. R. J. (2006). Reproductive ecology and behavior of Eleutherodactylus aureolineatus (Anura, Brachycephalidae) in the canopy of the Upper Amazon Basin, Ecuador. Phyllomedusa, 5, 135-143.

McCracken, S. F., \& Forstner, M. R. J. (2008). Bromeliad patch sampling technique for canopy herpetofauna in neotropical forests. Herpetological Review, 39, 170-174.

McNaughton, S. J. (1968). Structure and function in California grassland. Ecology, 49, 962-972. 
Mitchell, A. W., Secoy, K., \& Jackson, T. (2002). The global canopy handbook: Techniques of access and study in the forest roof. Oxford, Reino Unido: Global Canopy Programme.

Mondragón-Chaparro, D. M., \& Cruz-Ruiz, G. I. (2009). Seasonal variation of the macro-arthropod community associated to Tillandsia carlos-hankii (Bromeliaceae) in an oakpine forest in Oaxaca, Mexico. Brenesia, 70, 11-22.

Nadkarni, N. M. (1994). Diversity of species and interactions in the upper tree canopy of forest ecosystems. American Zoologist, 34, 70-78.

Palacios-Vargas, J. G. (1979). Los colémbolos (Ins.:Apter.) de suelo y hojarasca de epífitas. Folia Entomológica Mexicana, 42, 41-42.

Picado, C. (1913). Les bromeliacees epiphytes considerees comme milieu biologique. Bulletin Scientifique de la France et de la Belgique, 5, 215-360.

Ramírez, I. M., Carnevali, G., \& Chi, F. (2004). Guía ilustrada de las Bromeliaceae de la porción mexicana de la Península de Yucatán.., México D.F., México: Centro de Investigación Científica de Yucatán (CICY) A.C.

Ramírez-Villarroel, P. (1993). Estructura de las comunidades de peces en lagunas costeras de la isla de Margarita, Venezuela. Anales del Instituto de Ciencias del Mar y Limnología. UNAM (Universidad Nacional Autónoma de México). Recuperado de http://biblioweb.tic.unam.mx/cienciasdelmar/instituto/1994-1-2/articulo436.html

Richardson, B. A. (1999). The bromeliad microcosm and the assessment of faunal diversity in a neotropical forest. Biotropica, 31, 321-336.

Richardson, B. A., Rogers, C., \& Richardson, M. J. (2000). Nutrients, diversity, and community structure of two phytotelm systems lower montane forest, Puerto Rico. Ecological Entomology, 25, 348-356.

Rivalta, V., \& Díaz, L. M. (2003). Ranas de las ciudades. En Rodríguez, L. (Ed.). Anfibios y Reptiles de Cuba (pp. 44-49). Instituto de Ecología y Sistemática (IES), Cuba. Vaasa, Finlandia: UPC Print.

Rodríguez, A.. \& Alonso, R. (2003). Ranas arborícolas. En Rodríguez, L. (Ed.). Anfibios y Reptiles de Cuba (pp. 44-49). Instituto de Ecología y Sistemática (IES), Cuba. Vaasa, Finlandia: UPC Print.

Rodríguez, L. (ed.). 1999. The Iguanid lizards of Cuba. Gainesville, E.E.U.U.: University Press of Florida.
Rodríguez, L. 2003. Generalidades. En Rodríguez, L. (Ed.). Anfibios y Reptiles de Cuba (pp. 2-9). Instituto de Ecología y Sistemática (IES), Cuba. Vaasa, Finlandia: UPC Print.

Romero, G. Q. (2006). Geographic range, habitat, and host plants of bromeliad-living jumping spiders. Biotropica, $38,522-530$.

Romero, G. Q., Nomura, F., Gonçalves, A. Z., Días, N. Y. N., Mercier, H., Conforto, E. C., \& Rossa-Feres, D. C. (2010). Nitrogen Xuxes from treefrogs to tank epiphytic bromeliads: an isotopic and physiological approach. Oecologia, 162, 941-949.

Schiesari, L. C., Grillitsch, B., \& Vogt, C. (1996). Comparative morphology of phytotelmonous and pond-dwelling larvae of four neotropical treefrog species (Anura, Hylidae, Osteocephalus oophagus, O. taurinnus, Phrynohyas resinifictrix, P. venulosa. Alytes, 13, 109-139.

Schiesari, L. C., Gordo, M., \& Hödl, W. (2003). Treeholes as calling, breeding, and developmental sites for the Amazonian canopy frog, Phrynohyas resinifictrix (Hylidae). Copeia, 2003, 263-272.

Scott, N. J., \& Limerick, S. (1983). Reptiles and amphibians. En Janzen, D. (Ed.). Costa Rican Natural History (pp. 351374). Chicago, E.E.U.U.: University of Chicago Press.

Shaw, D. C. (2005).Vertical organization of canopy biota. En Lowman, M. D. \& Rinker, H.B. (Eds.). Forest canopies (pp. 73-96). San Diego California, E.E.U.U.: Elselvier Academic Press.

Smith, L. B., \& Downs, R. J. (1977). Tillandsioideae. Flora Neotropica, 14, 663-1492.

Smith, L. B., \& Downs, R. J. (1979). Bromelioideae. Flora Neotropica, 14, 1493-2142.

Stuntz, S. (2001). The influence of epiphytes on arthropods in thetropical forest canopy. (tesis doctoral) Universidad de Wüuzburg. Wüuzburg, Alemania.

Urquiola, A. J., González-Oliva, L., Novo, R., \& Acosta, Z. (2010). Libro rojo de la flora vascular de la provincia de Pinar del Río. Jardín Botánico de Pinar del Río, Cuba. España: Publicaciones Universidad de Alicante.

Wittman, P. K. (2000). The animal community associated with canopy bromeliads of the lowland peruvian amazon rain forest. Selbyana, 21, 48-51. 
